\title{
Correction to: Caveolin-1 Expression Together with VEGF can be a Predictor for Lung Metastasis and Poor Prognosis in Osteosarcoma
}

Fatma El-Zahraa Ammar Mohamed ${ }^{1}$ • El Zahraa Ibrahim Khalil ${ }^{1}$ • Nisreen D. M. Toni ${ }^{1}$

Published online: 21 January 2020

(C) Arányi Lajos Foundation 2020

Correction to: Pathology \& Oncology Research https://doi.org/10.1007/s12253-019-00755-5

The original version of this article unfortunately contained an error. The Tables 1 and 2 were missing in the published paper.

The missing Tables 1 and 2 are shown in the next page. The original article has been corrected.

The online version of the original article can be found at https://doi.org/ 10.1007/s12253-019-00755-5

El Zahraa Ibrahim Khalil

zahraa333eg@gmail.com

Pathology Department, Faculty of Medicine, Minia University,

Minia, Egypt 
Table 1 Relationship between Caveolin1 expression and different clinicopathological parameters in OS
Table 2 Relationship between VEGF expression and different clinicopathological parameters in OS

\begin{tabular}{lllll}
\hline Clinicopathological variables & $\begin{array}{l}\text { Total } \\
N=66\end{array}$ & $\begin{array}{l}\text { High Cav-1 } \\
N=13\end{array}$ & $\begin{array}{l}\text { Low Cav-1 } \\
N=53\end{array}$ & $P$ value \\
\hline $\begin{array}{l}\text { Age } \\
\text { Sex }\end{array}$ & & $14 \pm 3.15$ & $14 \pm 1.96$ & 0.995 \\
Male & 42 & $11(26.2 \%)$ & $31(73.8 \%)$ & 0.074 \\
Female & 24 & $2(8.3 \%)$ & $22(91.7 \%)$ & \\
Histologic subtype & 24 & 0 & 24 & $\mathbf{P}<\mathbf{0 . 0 0 0 1}$ \\
Chondroblastic & 36 & 13 & 23 & \\
Osteoblastic & 6 & 0 & 6 & 0.705 \\
Fibroblastic & 48 & $10(20.8 \%)$ & $38(79.2 \%)$ & $15(83.3 \%)$ \\
Stage & 18 & $3(16.7 \%)$ & & \\
Stage II & & $11(20.4 \%)$ & $43(79.6 \%)$ & 0.770 \\
Stage III & 54 & $2(16.7 \%)$ & $10(83.3 \%)$ & \\
Site & 12 & &
\end{tabular}

\begin{tabular}{lllll}
\hline Clinicopathological variables & $\begin{array}{l}\text { Total } \\
N=66\end{array}$ & $\begin{array}{l}\text { Positive VEGF } \\
N=51\end{array}$ & $\begin{array}{l}\text { Negative VEGF } \\
N=15\end{array}$ & $P$ value \\
\hline Age & & $14.4 \pm 2.19$ & $12.60 \pm 4.27$ & $\mathbf{0 . 0 3 1}$ \\
Sex & 42 & $30(71.4 \%)$ & $12(28.6 \%)$ & 0.121 \\
Male & 24 & $21(87.5 \%)$ & $3(12.5 \%)$ & \\
Female & 24 & $18(75 \%)$ & $6(25 \%)$ & 0.143 \\
Histologic subtype & 36 & $30(83.3 \%)$ & $6(16.7 \%)$ & \\
Chondroblastic & 6 & $3(50 \%)$ & $3(50 \%)$ & \\
Osteoblastic & 48 & $33(68.75 \%)$ & $15(31.25 \%)$ & $\mathbf{P}<\mathbf{0 . 0 0 1}$ \\
Fibroblastic & 18 & $18(100 \%)$ & 0 & \\
Stage & & $39(72.2 \%)$ & $15(27.8 \%)$ & $\mathbf{0 . 0 2 8}$ \\
Stage II & 54 & $12(100 \%)$ & 0 & \\
Stage III & 12 & & \\
Site & & & & \\
Extremities & & & & \\
Metastatic lung OS & & & & \\
\hline
\end{tabular}

